\title{
Luminescence and Electronic Excitations in $\mathrm{KBe}_{2} \mathrm{BO}_{3} \mathrm{~F}_{2}$ Crystals
}

\author{
I. N. Ogorodnikov ${ }^{a}$, V. A. Pustovarov ${ }^{a}$, S. A. Yakovlev ${ }^{a}$, L. I. Isaenko ${ }^{b}$, and S. A. Zhurkov ${ }^{b}$ \\ ${ }^{a}$ Yeltsin Ural Federal University (Ural State Technical University-UPI), \\ ul. Mira 19, Yekaterinburg, 620002 Russia \\ *e-mail: igor.ogorodnikov@bk.ru \\ ${ }^{b}$ Sobolev Institute of Geology and Mineralogy, Siberian Branch of the Russian Academy of Sciences, \\ pr. Akademika Koptyuga 3, Novosibirsk, 630090 Russia \\ Received October 4, 2011
}

\begin{abstract}
This paper reports on a study of the dynamics of electronic excitations in $\mathrm{KBe}_{2} \mathrm{BO}_{3} \mathrm{~F}_{2}(\mathrm{KBBF})$ crystals by low-temperature luminescent vacuum ultraviolet spectroscopy with nanosecond time resolution under photoexcitation by synchrotron radiation. The first data have been obtained on the kinetics of photoluminescence (PL) decay, time-resolved PL spectra, time-resolved PL excitation spectra, and reflection spectra at $7 \mathrm{~K}$; the estimation has been performed for the band gap $E_{\mathrm{g}}=10.6-11.0 \mathrm{eV}$; the predominantly excitonic mechanism for PL excitation at $3.88 \mathrm{eV}$ has been identified; and defect luminescence bands at 3.03 and $4.30 \mathrm{eV}$ have been revealed. The channels of generation and decay of electronic excitations in KBBF crystals have been discussed.
\end{abstract}

DOI: $10.1134 / \mathrm{S} 106378341204018 \mathrm{X}$

\section{INTRODUCTION}

$\mathrm{KBe}_{2} \mathrm{BO}_{3} \mathrm{~F}_{2}$ (KBBF) crystals were first synthesized in 1968 (Novosibirsk) [1, 2]. Only a quarter of a century later, however, progress reached in growth technology made this crystal widely available for studies and applications. The main difficulty encountered in its use is associated with the layered structure of this optical material, which decomposes rapidly at a high temperature. As a result, the thickness of grown crystals does not usually exceed $2 \mathrm{~mm}$ [3]. KBBF has gained extreme popularity after its remarkable nonlinear optical properties in the short-wavelength region of the spectrum became known, to wit, the nonlinear optical coefficient is $d=0.49 \mathrm{pm} / \mathrm{V}$, second harmonic generation efficiency reaches $26.1 \%$, and the threshold of damage by laser radiation $(\lambda=1064 \mathrm{~nm}, \tau=$ $10 \mathrm{~ns})$ is in excess of $40 \mathrm{GW} / \mathrm{cm}^{2}[3,4]$. By now, one successfully obtains on a KBBF crystal the sixth harmonic $(\lambda=177.3 \mathrm{~nm})$ of $\mathrm{Nd}: \mathrm{YVO}_{4}$ or $\mathrm{Nd}$ : YAG lasers [3-7].

KBBF crystals belong to trigonal crystal system (space group 232 ). The unit cell contains three formula units (27 atoms) with the lattice parameters $a=$ $b=0.4427$ (4) nm, $c=1.8744(9) \mathrm{nm}, \alpha=\beta=90^{\circ}, \gamma=$ $120^{\circ}$ [4]. The crystallographic structure of KBBF is formed by the anion groups $\left[\mathrm{BO}_{3}\right]^{3-}$ and $\left[\mathrm{BeFO}_{3}\right]^{5-}$, with the empty spaces occupied by $\mathrm{K}^{+}$cations. The spatial structure consists of nearly planar $\mathrm{Be}_{2} \mathrm{BO}_{3}$ sixatom rings. In each ring, two beryllium atoms are bound to two additional fluorine atoms located beyond the ring plane. Neighboring rings make up a continuous boron-oxygen framework [8,9]. At room temperature, the KBBF crystal is chemically stable and nonhygroscopic. Its specific weight is $4.550 \mathrm{~g} / \mathrm{cm}^{3}$ [10].

KBBF is an optically negative uniaxial crystal with an optical transmission band of 153-366 nm. The refractive indices at the wavelength of $1064 \mathrm{~nm}$ are $n_{0}=1.477$ and $n_{e}=1.400$ [4]. The broad band of optical transparency and good nonlinear optical properties permit one to class KBBF among the shortestwave nonlinear optical crystals which have high engineering potential in the ultraviolet (UV) and vacuum ultraviolet (VUV) spectral regions. At the same time, it is the crystallographic structure that accounts for the specific features of the electronic structure and affects transfer of electronic excitation (EE) energy and the mechanisms responsible for defect formation in KBBF. These aspects require a comprehensive investigation of KBBF by the methods employed in spectroscopy of the solid state. There is a lot of publications presently that deal with the technology of growing and studies of the nonlinear optical properties of KBBF (see, e.g., [11-13]). We are, however, unaware of any papers dealing with investigation of the luminescence characteristics of KBBF.

The present work was aimed at studying the generation and evolution of EEs in KBBF crystals by time resolved, low-temperature VUV luminescence spectroscopy performed under selective excitation with synchrotron radiation. 


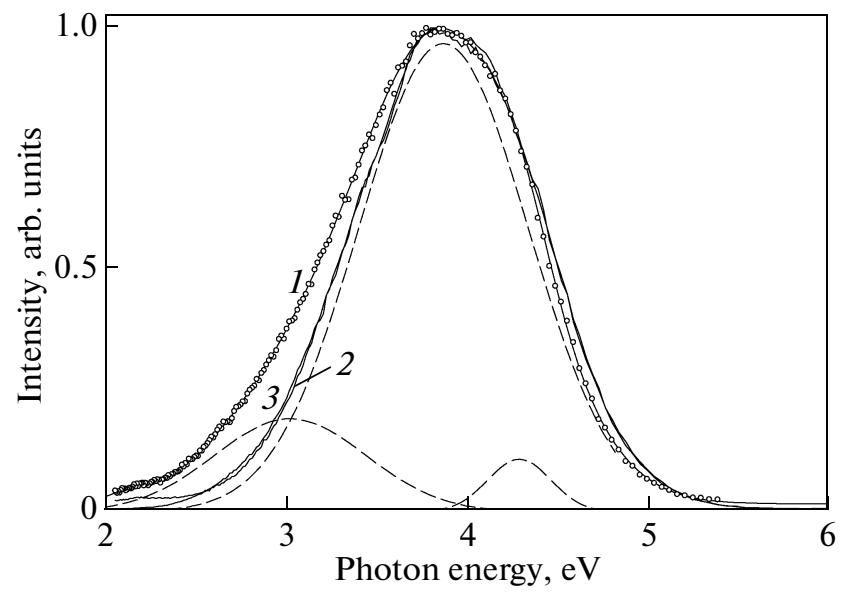

Fig. 1. Spectrum of the time-integrated PL component of the KBBF crystal at $T=7 \mathrm{~K}$ and $E_{\mathrm{ex}}=$ (1) 6.5, (2) 8.7, and (3) $11.1 \mathrm{eV}$. The circles and solid lines are experimental data, and dashed lines visualize the Gaussian constituent bands derived by resolving curve 1 .

\section{EXPERIMENTAL DETAILS}

We studied KBBF crystals of optical quality grown at the Institute of Geology and Mineralogy of the Siberian Branch of the Russian Academy of Sciences (Novosibirsk) by spontaneous crystallization in a platinum crucible, $40 \mathrm{~mm}$ in diameter and $50 \mathrm{~mm}$ high, in a pit furnace with ohmic heating [14]. The starting reagents were $\mathrm{K}_{2} \mathrm{CO}_{3}, \mathrm{BeO}, \mathrm{H}_{3} \mathrm{BO}_{3}, \mathrm{KBF}_{4}$, and $\mathrm{KF}$ of 99.999\% purity. The KBBF compound was prepared by solid-phase synthesis

$$
\begin{gathered}
\mathrm{K}_{2} \mathrm{CO}_{3}+8 \mathrm{BeO}+2 \mathrm{H}_{3} \mathrm{BO}_{3}+2 \mathrm{KBF}_{4} \\
=4 \mathrm{KBe}_{2} \mathrm{BO}_{3} \mathrm{~F}_{2}+\mathrm{CO}_{2}+3 \mathrm{H}_{2} \mathrm{O} .
\end{gathered}
$$

The salt powders were mixed thoroughly and placed into a platinum glass, which was subsequently heated for $12 \mathrm{~h}$ up to a temperature of $900^{\circ} \mathrm{C}$. The furnace was maintained at this temperature for $4 \mathrm{~h}$ and turned off thereafter. The extent to which the reaction has progressed was checked by weighing, and identification of the powder thus prepared with KBBF was performed by testing for generation of the second laser harmonic with the wavelength $\lambda=1064 \mathrm{~nm}$. The powder thus produced was subsequently used as the starting reagent for growing KBBF crystal.

A mixture of $\mathrm{KF}$ with $\mathrm{H}_{3} \mathrm{BO}_{3}$ was used as solvent. The starting composition of the $\mathrm{KBBF}, \mathrm{KF}, \mathrm{H}_{3} \mathrm{BO}_{3}$ mixture was $1.0: 4.5: 2$. After homogenization run at $750^{\circ} \mathrm{C}$ for several hours, a seed was dropped into the melt, and the temperature was lowered down to the equilibrium point at about $710-720^{\circ} \mathrm{C}$. The growth proceeded with the temperature reduced with a rate that increased gradually from $0.5^{\circ} \mathrm{C}$ to $5.0^{\circ} \mathrm{C}$ per day. In the time of growth, the temperature decreased by $55-60^{\circ} \mathrm{C}$.
The plates thus produced, $20 \times 20 \mathrm{~mm}$ in size, consisted of large blocks up to $2.0 \mathrm{~mm}$ thick. The samples intended for spectroscopic studies were plane-parallel transparent plates measuring $6 \times 5 \times 1.5 \mathrm{~mm}$.

The time-resolved photoluminescence (PL) spectra in the 1.2-6.2 eV interval, time-resolved PL excitation spectra $(3.7-21.0 \mathrm{eV})$, spectra of reflection at an angle of $17^{\circ}$, and the PL decay kinetics measured with subnanosecond time resolution were obtained under selective excitation by synchrotron radiation (SR) at the SUPERLUMI station [15] of HASYLAB laboratory. The SR pulses of the DORIS storage ring had a Gaussian shape $(\mathrm{FWHM}=130 \mathrm{ps})$ produced with a repetition period of $96 \mathrm{~ns}$. The measurements were performed at a temperature of $7 \mathrm{~K}$ with a gas-flow helium cryostat providing a vacuum of not worse than $7 \times 10^{-10}$ Torr. The time-resolved spectra were recorded in a time window of thickness $\Delta t$ with a delay of $\delta t$ relative to the SR excitation pulse. In this study, recording was done simultaneously in two independent windows with the parameters $\delta t_{1}=0.5 \mathrm{~ns}, \Delta t_{1}=$ $2.3 \mathrm{~ns}$ (fast component) and $\delta t_{2}=14 \mathrm{~ns}, \Delta t_{2}=58 \mathrm{~ns}$ (slow component). One recorded also a PL spectrum without time resolution (time-integrated component). For selective excitation of PL by synchrotron radiation in the 3.7-21.0-eV interval one used a 2-m-long vacuum monochromator equipped with an $\mathrm{Al}$ grating (spectral resolution $0.32 \mathrm{~nm}$ ). The PL excitation spectra were normalized to equal number of photons incident on the sample. PL spectra in the 1.5-6.2-eV interval were measured with a $0.3-\mathrm{m}$ (ARC Spectra Pro-308i), R6358P (Hamamatsu) photomultiplier tube, or CCD camera. PL spectra were nor corrected for the spectral sensitivity of the optical path. The fast components in the PL decay kinetics were analyzed with the use of the convolution integral, with due allowance for the instrument contour of the excitation pulse (FWHM of about $300 \mathrm{ps}$ ).

\section{RESULTS OF THE EXPERIMENT}

The low-temperature luminescence of KBBF crystals was studied within a broad spectral region, from 1.4 to $6.2 \mathrm{eV}$, under selective excitation by photons of various energies $E_{\mathrm{ex}}$ ranging from 3.7 to $21.0 \mathrm{eV}$. At $7 \mathrm{~K}$, the PL spectrum of the KBBF crystal is dominated by a broad band of maxima in the $3.6-4.2-\mathrm{eV}$ interval (Fig. 1). The position of the maximum and the spectral contour of the band depend on the exciting photon energy, which suggests that the PL curve observed is not a single band. Table 1 presents the characteristics of the decomposition of the timeresolved PL spectra of the KBBF crystal measured at $T=7 \mathrm{~K}$ and $E_{\text {ex }}=6.5,8.7$, and $11.1 \mathrm{eV}$. As seen from the table, all the changes in the profile of the observed PL band originate from the variation of the amplitudes of the three main Gaussian-shaped constituent bands peaking at $3.03,3.88$, and $4.30 \mathrm{eV}$. In what follows, the 
amplitudes of the constituent bands are referred to the intensity of the observed PL band normalized against unity.

The band at $3.03 \mathrm{eV}$ becomes excited most efficiently in the crystal transparency region, where its relative amplitude reaches as high as 0.20 . In the region of fundamental absorption, the relative amplitude of this band decreases approximately by a factor 20 for the fast component, and by 4-5 times, for the time-integrated component. Figure 2 presents a PL spectrum measured with a CCD camera. The lowenergy band peaking at about $3 \mathrm{eV}$ stands out here more clearly due to the differences in spectral sensitivity between the PM tube and the CCD camera in this spectral region.

The two other constituent bands become excited efficiently at all the excitation energies used. Interestingly, the band at $3.88 \mathrm{eV}$ is dominant, with a relative amplitude of $0.91-0.99$, while that of the $4.30-\mathrm{eV}$ band is $0.10-0.20$ (see table).

Besides the three main constituent bands, two additional low-intensity bands were revealed in decomposition of the PL spectra. The band at $2.2 \mathrm{eV}$ $(\mathrm{FWHM}=0.2 \mathrm{eV})$ having a relative amplitude of $0.01-0.02$ is excited only at $E_{\mathrm{ex}}=6.5$ and $8.7 \mathrm{eV}$. The band at $4.5 \mathrm{eV}(\mathrm{FWHM}=0.8 \mathrm{eV})$ with a relative amplitude of $0.03-0.05$ becomes excited at $E_{\text {ex }}=$ $8.7 \mathrm{eV}$ only.

Figure 3 displays excitation spectra of the timeintegrated PL components at $4.1 \mathrm{eV}$ and a reflection spectrum of the KBBF crystal measured at $7 \mathrm{~K}$. The PL excitation spectrum obtained at $E_{\mathrm{m}}=4.1 \mathrm{eV}$ permits isolation of three regions. In the $5.0-8.0 \mathrm{eV}$ region, the excitation spectrum is only weakly structured, and the excitation efficiency is fairly low and does not exceed $10 \%$ of the observed maximum. In the 8.0 $-10.0 \mathrm{eV}$ region, the PL excitation efficiency grows

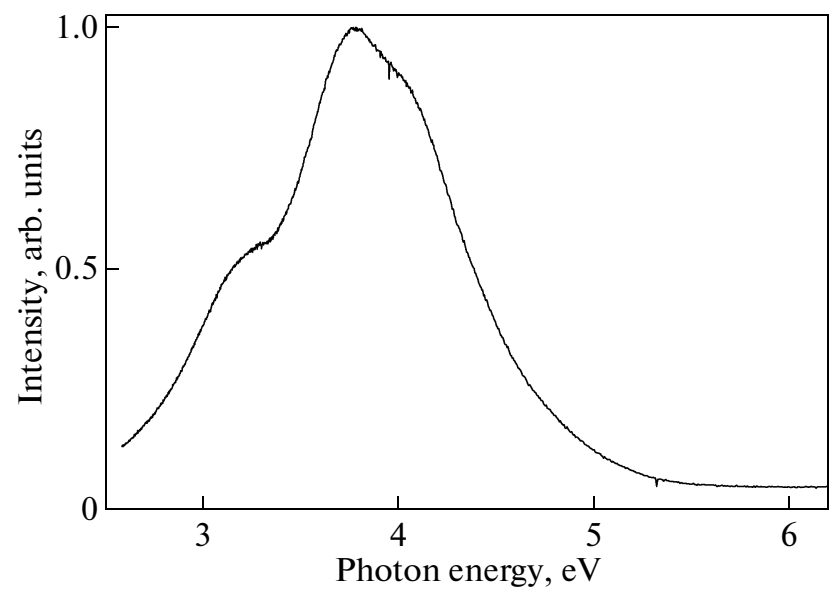

Fig. 2. PL spectrum of the KBBF crystal at $T=7 \mathrm{~K}$ and $E_{\text {ex }}=11.1 \mathrm{eV}$, measured in the $2.5-6.2-\mathrm{eV}$ interval by means of a CCD camera.
Parameters of time-resolved PL bands of the KBBF crystal at $7 \mathrm{~K}$

\begin{tabular}{c|c|c|c|c}
\hline \multirow{2}{*}{$\begin{array}{c}E_{m}, \mathrm{eV} \\
(\Delta E, \mathrm{eV})\end{array}$} & \multirow{2}{*}{$\begin{array}{c}\text { Gaussian } \\
\text { component }\end{array}$} & \multicolumn{3}{|c}{$E_{\mathrm{ex}}, \mathrm{eV}$} \\
\cline { 3 - 5 } & & 6.5 & 8.7 & 11.1 \\
\hline 3.03 & $I_{\mathrm{F}}$ & 0.21 & 0.01 & 0.01 \\
$(0.99)$ & $I_{\mathrm{S}}$ & 0.17 & - & - \\
& $I_{\mathrm{T}}$ & 0.19 & 0.04 & 0.05 \\
3.88 & $I_{\mathrm{F}}$ & 0.92 & 0.94 & 0.97 \\
$(1.10)$ & $I_{\mathrm{S}}$ & 0.91 & 0.96 & 0.97 \\
& $I_{\mathrm{T}}$ & 0.95 & 0.99 & 0.99 \\
4.30 & $I_{\mathrm{F}}$ & 0.21 & 0.13 & 0.09 \\
$(0.42)$ & $I_{\mathrm{S}}$ & 0.09 & 0.14 & 0.12 \\
& $I_{\mathrm{T}}$ & 0.11 & 0.09 & 0.11 \\
\hline
\end{tabular}

Note: $E_{m}, \Delta E$, and $I_{v}$ are the position of the maximum, FWHM, and amplitude of the Gaussian constituent with respect to that of the observed PL band normalized to unity; $v$ stands for the fast (F), slow (S), and time-integrated (T) components of PL; $E_{\mathrm{ex}}$ is the energy of exciting photons.

strongly by about a factor 20 and reveals a sharp peak with a maximum at $8.7 \mathrm{eV}$. At excitation energies $E_{\mathrm{ex}}>$ $10.0 \mathrm{eV}$, this peak overlaps partially with another one at $10.6 \mathrm{eV}$, after which one observes a smooth falloff of the PL excitation efficiency down to $40 \%$ for the fast and time-integrated components, and to $20 \%$ for the

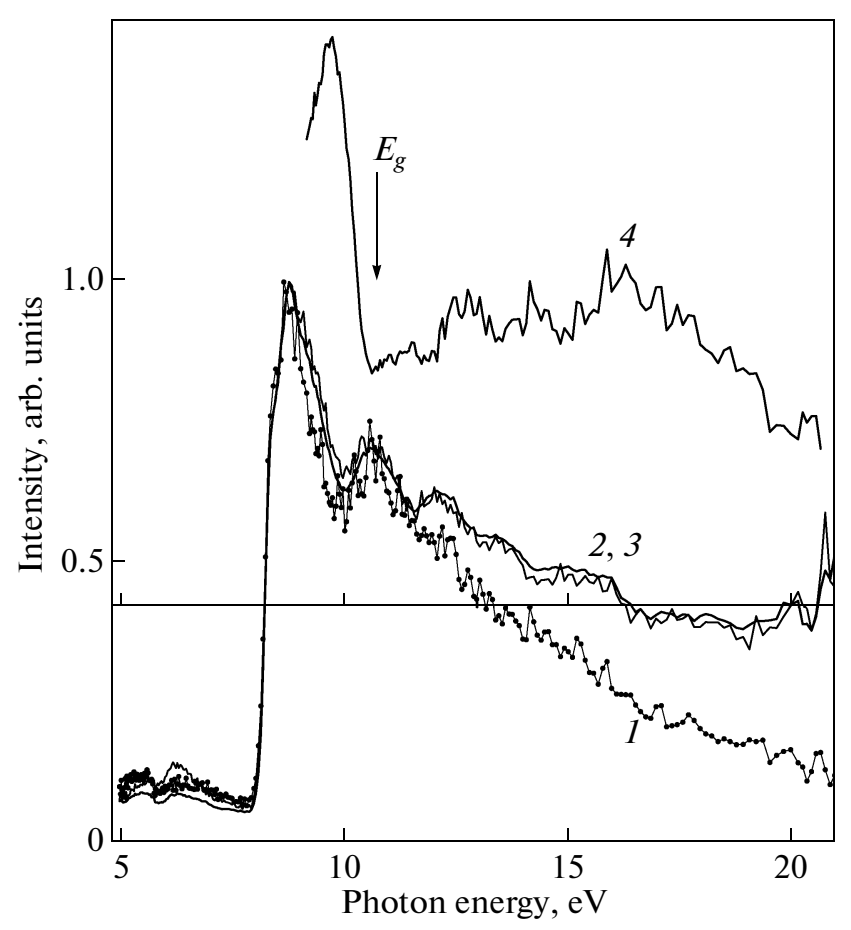

Fig. 3. Excitation spectrum of the (1) slow, (2) fast, and (3) time-integrated PL components at $E_{\mathrm{m}}=4.1 \mathrm{eV}$, and reflection spectrum (4) for a $\mathrm{KBBF}$ crystal at $T=7 \mathrm{~K}$. The reflection spectrum is offset vertically for clarity. 


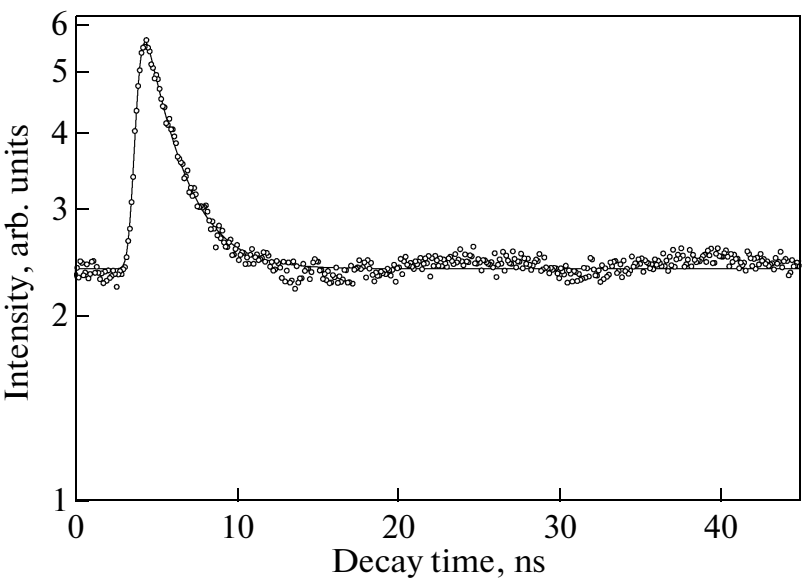

Fig. 4. PL decay kinetics of the KBBF crystal measured at $7 \mathrm{~K}$ in the luminescence band at $4.1 \mathrm{eV}$ with the exciting photon energy of $11.1 \mathrm{eV}$. Solid line is the approximation, open circles are experimental data.

slow PL component. Time-resolved excitation spectra of the other PL bands were also measured, but they all turned out to be identical with the one considered above and are not displayed in the Figure. The lowtemperature reflection spectrum of KBBF is dominated by a peak at $9.7 \mathrm{eV}$. In the $9.8-10.6 \mathrm{eV}$ region, the reflection intensity drops sharply by about one half, to transform subsequently in the energy region extending to $21 \mathrm{eV}$ to a weakly structured spectrum.

The PL kinetics decay of KBBF at $7 \mathrm{~K}$ was measured for different values of $E_{\mathrm{m}}$ ranging from 2.9 to $4.4 \mathrm{eV}$ under selective excitation by photons with energies $E_{\text {ex }}=8.7$ or $11.1 \mathrm{eV}$. In all the cases studied, the decay kinetics was found to follow identical profiles. Figure 4 exemplifies the PL decay kinetics measured for $E_{\mathrm{m}}=4.1 \mathrm{eV}$ and $E_{\mathrm{ex}}=11.1 \mathrm{eV}$. The kinetics is dominated by a fast exponential component with a time constant $\tau=2 \mathrm{~ns}$, with superposing micro- and millisecond-range components seen as a pedestal in our measurements. The ratio of the exponent amplitude to the height of the pedestal is about 0.96 .

\section{DISCUSSION OF THE RESULTS}

The quantum-chemical calculations of the electronic structure of $\mathrm{KBBF}[11,16,17]$ suggest that the valence band (VB) of $\mathrm{KBBF}$ is about $10 \mathrm{eV}$ in width and consists of two subbands derived primarily from the oxygen $2 p$ orbitals. The VB top is practically free of hybridization between the boron and oxygen orbitals, with the potassium $p$ orbitals becoming evident $10 \mathrm{eV}$ below the VB top. The bottom of the conduction band is derived from hybridized orbitals of boron and oxygen, whereas the beryllium $2 p$ orbitals contribute moderately to higher-lying states of the conduction band. The large width of the KBBF band gap should be attributed to mixing of the fluorine and oxygen $2 p$ orbitals [11]. The value $E_{\mathrm{g}}=5.259 \mathrm{eV}$ calculated by the DFT method [11] appears to be an underestimation, because experimental measurements $[3,4]$ show the optical transparency band of KBBF to extend to $153 \mathrm{~nm}(8.1 \mathrm{eV})$. The lowest-energy EEs in KBBF crystals are produced by electron transfer from states at the valence band top to those of the conduction band bottom, i.e., in electron transitions within an anion group.

In the reflection spectrum of KBBF (Fig. 3), the lowest-energy dominant peak at $9.7 \mathrm{eV}$ falls into the region of a comparatively narrow and intense band peaking at $8.7 \mathrm{eV}$ in the PL excitation spectrum and, thus, can be identified with creation in the crystal of unrelaxed EEs. A comparison of the reflection spectra of KBBF (Fig. 3) with the experimental and theoretical data available for other borates (for instance, $\left.\mathrm{Li}_{2} \mathrm{BO}_{4}[18,19]\right)$ suggests a well substantiated estimate for the KBBF band-gap width of $E_{\mathrm{g}}=10.6-11.0 \mathrm{eV}$.

Photoluminescence is most efficiently excited in the region of fundamental absorption of the crystal. The sharp monotonic growth of the PL excitation efficiency observed to occur in the $8.0-8.5 \mathrm{eV}$ interval correlates with the increase of optical absorption in the region of the long-wavelength fundamental absorption edge. In full agreement with the Bouguer-Lambert law, as the excitation energy continues to increase, the competing process of decreasing VUV photon free path initiating growth of the fraction of surface energy losses becomes dominant, which gives rise to the highenergy slope of the excitation peak at $8.8-10.0 \mathrm{eV}$. The maximum of the peak at $8.7 \mathrm{eV}$ signifies a balance reached between these competing processes. The shape of the KBBF PL excitation spectrum in the region of $8.0-10.0 \mathrm{eV}$ suggests the excitonic mechanism of energy transfer in this crystal, a feature characteristic of systems in which luminescence is of excitonic origin, or where excitons act as an intermediate link in energy transfer to luminescence centers.

Further increase of the energy of exciting photons provokes decay of the excitation efficiency. Above $10 \mathrm{eV}$, the structure of the reflection spectrum behaves in opposite phase to that of the excitation spectrum of the fast and time-integrated PL components (Fig. 3). This may be considered as an indirect evidence for the formation in KBBF of mobile EEs in photoexcitation of the crystal in the region of fundamental absorption. Their efficient migration may end up in nonradiative relaxation at surface defects, thus accounting for surface energy losses.

For $E_{\mathrm{ex}}>E_{\mathrm{g}}$, exciton formation is paralleled by the appearance of separated charge carriers, namely, of holes in the valence band and of the corresponding number of electrons in the conduction band. If the energy of the exciting photons $E_{\text {ex }}$ only slightly exceeds $E_{\mathrm{g}}$, the kinetic energy of the electron in the conduction band will be not high enough to leave the potential well created by the hole Coulomb potential. In this case, 
the most probable process is recombination in genetic electron-hole pairs [20-22]. The pattern of the excitation spectrum of the slow KBBF PL component in the $10.0-21.0 \mathrm{eV}$ region suggests that electron-hole recombination occurs predominantly in genetic electron-hole pairs (Fig. 3). As the energy $E_{\text {ex }}$ increases, the kinetic energy of an electron in the conduction band and, accordingly, the probability of separation of electron-hole pairs increases too. In this case the separated charge carriers can be captured and/or recombine at local defect levels. And it is with this case that we identify the excitation spectra of the fast and timeintegrated KBBF PL components in Fig. 3.

In both cases, defects must be involved, because the fact itself of PL photoexcitation in the crystal transparency band excludes the possibility of EE self-trapping. It is known [23] that if viewed in terms of EE dynamics, lattice defects can be formally divided into two categories, namely, defects favoring EE localization (localization centers) and those transforming the EE energy to the excited state of the defect (transformation centers). The cross sections of exciton trapping by these centers differ in their dependence on energy. At energies below $E_{\mathrm{g}}$, the cross section for trapping by a localization center (point defect or an insignificant lattice distortion) increases with decreasing energy and reaches a maximum near the bottom of the exciton band. The exciton trapping cross section for a transformation center (self-trapped hole, charged or neutral impurity) exhibits the opposite behavior with energy [23]

After photocreation of the exciton at energies below $E_{\mathrm{g}}$, it relaxes following the dispersion curve to the lower part of the exciton band, where it becomes trapped by localization centers in interaction with the vibronic states. In this part of the exciton band, the competition in trapping by the transforming centers is suppressed. The exciton trapped by a localizing center is equivalent to an exciton created in the very beginning at the exciton band bottom. This is why the relaxation channel culminating in annihilation of a trapped exciton is dominant at excitation energies below $E_{\mathrm{g}}$ (Fig. 3).

The situation is different at energies above $E_{\mathrm{g}}$. A significant part of the EE energy can become lost in nonradiative relaxation at luminescence quenching centers (for instance, uncontrollable impurities) or as a result of surface losses. Because the probability of recombination of band electrons and holes is fairly small, the main channel will be here recombination of the band electron (or hole) with a trapped carrier of opposite sign. This brings about formation of a localized EE, which relaxes via interaction with the vibronic states. In the top part of the exciton band, this relaxation channel interferes weakly with states of a free exciton, which results in a high probability of trapping of the localizing EE by transformation centers. This culminates in a drop of the luminescence yield of the trapped or self-trapped EEs at excitation energies above $E_{\mathrm{g}}$ (Fig. 3 ).

These processes may result in radiative annihilation of the exciton, exciton energy transfer to a luminescence center, or migration of the exciton with subsequent nonradiative relaxation. Additional studies would, however, be needed to reliably identify the luminescence centers in KBBF. As follows from general considerations, the contribution of the excitonic mechanism to energy transfer can be significant only in the case of high concentrations of the above defects, when the probability of exciton formation in the immediate vicinity of these centers increases. At the same time, detailed discussion of this problem at this stage would be hardly possible, because there are only a few studies of macroscopic defects in a real KBBF structure $[24,25]$, and as for experimental works on point defects in KBBF, they are absent altogether.

\section{CONCLUSIONS}

Thus, this paper presented results of the first study of KBBF crystals by low-temperature, luminescentoptical vacuum ultraviolet spectroscopy performed with nanosecond time resolution. A combined analysis of these first experimental data on the photoluminescence decay kinetics, time-resolved PL spectra, time-resolved PL excitation spectra and reflection spectra measured at $7 \mathrm{~K}$ has produced an estimate of the band gap width $E_{\mathrm{g}}=10.6-11.0 \mathrm{eV}$, identified the predominantly excitonic mechanism of PL excitation at $3.88 \mathrm{eV}$, and resolved the defect luminescence bands at 3.03 and $4.30 \mathrm{eV}$. The channels of creation and decay of electronic excitations in KBBF crystals have been discussed.

\section{ACKNOWLEDGMENTS}

This study was supported in part by HASYLAB DESY (project no. 20080019).

\section{REFERENCES}

1. L. R. Batsanova, V. A. Egorov, and A. V. Nikolaev, Dokl. Akad. Nauk SSSR 178, 1317 (1968).

2. L. P. Solov'eva and V. V. Bakakin, Sov. Phys. Crystallogr. 15 (5), 802 (1970).

3. C. T. Chen, Opt. Mater. 26, 425 (2004).

4. C. T. Chen, Z. Xu, D. Deng, J. Zhang, G. K. L. Wong, B. Wu, N. Ye, and D. Tang, Appl. Phys. Lett. 68, 2930 (1996).

5. T. Togashi, T. Kanai, T. Sekikawa, S. Watanabe, C. T. Chen, C. Zhang, Z. Xu, and J. Wang, Opt. Lett. 28, 254 (2003).

6. Z.-G. Hu, M. Yoshimura, Y. Mori, and T. Sasaki, J. Cryst. Growth 275, 232 (2005).

7. C. Li, Y. Zhou, N. Zong, Z. Xu, X. Wang, and Y. Zhu, Chin. Opt. Lett. 7, 621 (2009). 
8. L. Mei, X. Huang, Y. Wang, Q. Wu, B. C. Wu, and C. T. Chen, Z. Kristallogr. 210, 93 (1995).

9. L. Mei, Y. Wang, C. T. Chen, and B. Wu, J. Appl. Phys. 74, 7014 (1993).

10. C. D. McMillan and J. W. Kolis, J. Cryst. Growth 310, 2033 (2008).

11. Z. Lin, Z. Wang, C. T. Chen, S. K. Chen, and M.-H. Lee, Chem. Phys. Lett. 367, 523 (2003).

12. K. Hussain and P. Kumbhakar, Brazilian J. Phys. 36, 1281 (2006).

13. T. Sasaki, Y. Mori, M. Yoshimura, Y. K. Yap, and T. Kamimura, Mater. Sci. Eng., R. 30, 1 (2000).

14. L. I. Isaenko and A. P. Yelisseyev, Chem. Sustainable Dev. 8, 213 (2000).

15. G. Zimmerer, Nucl. Instrum. Methods Phys. Res., Sect. A 308, 178 (1991).

16. C. T. Chen, N. Ye, J. Lin, J. Jiang, W. R. Zeng, and B. C. Wu, Adv. Mater. (Weinheim) 11, 1071 (1999).

17. Z. S. Lin, J. Lin, Z. Z. Wang, Y. C. Wu, N. Ye, C. T. Chen, and R. K. Li, J. Phys.: Condens. Matter 13, R369 (2001).

18. A. Yu. Kuznetsov, L. I. Isaenko, A. V. Kruzhalov, I. N. Ogorodnikov, and A. B. Sobolev, Phys. Solid State 41 (1), 48 (1999).
19. I. N. Ogorodnikov, V. A. Pustovarov, A. V. Kruzhalov, L. I. Isaenko, M. Kirm, and G. Zimmerer, Phys. Solid State 42 (3), 464 (2000).

20. A. N. Vasil'ev, V. V. Mikhailin, and I. V. Ovchinnikova, Izv. Akad. Nauk SSSR, Ser. Fiz. 49, 2044 (1985).

21. J. Becker, A. N. Belsky, D. Bouttet, C. Dujardin, A. V. Gektin, A. Hopkirk, S. N. Ivanov, I. A. Kamenskikh, N. Y. Kirikova, V. Klimenko, V. N. Kolobanov, V. N. Makhov, P. Martin, V. V. Mikhailin, I. H. Munro, C. Mythen, P. A. Orekhanov, C. Pedrini, A. Schröder, D. A. Shaw, N. Shiran, I. N. Shpinkov, A. N. Vasil'ev, and G. Zimmerer, in Proceedings of the International Conference on Inorganic Scintillators and Their Applications (SCINT 95), Delft, The Netherlands, August 28September 1, 1995 (Delft University Press, Delft, The Netherlands, 1996), p. 118.

22. A. N. Vasil'ev, IEEE Trans. Nucl. Sci. 55, 1054 (2008).

23. M. A. Elango, Elementary Inelastic Radiation-Induced Processes (Nauka, Moscow, 1988; American Institute of Physics, New York, 1991).

24. Z. S. Lin, L. Bai, L. Liu, M. H. Lee, J. Xu, X. Wang, and C. T. Chen, J. Appl. Phys. 109, 073721 (2011).

25. J. Yu, L. Liu, X. Wang, H. Zhou, X. He, C. Zhang, W. Zhou, and C. T. Chen, J. Cryst. Growth 318, 621 (2011).

Translated by G. Skrebtsov 